\title{
On the regularity of the solutions of Dirichlet optimal control problems in polygonal domains*
}

\author{
Thomas Apel $^{\dagger} \quad$ Mariano Mateos ${ }^{\ddagger} \quad$ Johannes Pfefferer ${ }^{\S}$ \\ Arnd Rösch
}

October 3, 2018

\begin{abstract}
A linear quadratic Dirichlet control problem posed on a possibly non-convex polygonal domain is analyzed. Detailed regularity results are provided in classical Sobolev (Slobodetskiū) spaces. In particular, it is proved that in the presence of control constraints, the optimal control is continuous despite the non-convexity of the domain.
\end{abstract}

Key Words optimal control, boundary control, Dirichlet control, non-convex polygonal domain

AMS subject classification $\quad 65 \mathrm{~N} 30,65 \mathrm{~N} 15,49 \mathrm{M} 05,49 \mathrm{M} 25$

\section{Introduction}

The investigation of optimal control problems with partial differential equations has been of increasing interest in the last decades. In this paper we will study the control problem

$$
(\mathrm{P})\left\{\begin{array}{l}
\min J(u)=\frac{1}{2} \int_{\Omega}\left(S u(x)-y_{\Omega}(x)\right)^{2} d x+\frac{\nu}{2} \int_{\Gamma} u^{2}(x) d \sigma(x) \\
\text { subject to }(S u, u) \in H^{1 / 2}(\Omega) \times L^{2}(\Gamma), \\
u \in U_{a d}=\left\{u \in L^{2}(\Gamma): a \leq u(x) \leq b \quad \text { for a.a. } \quad x \in \Gamma\right\},
\end{array}\right.
$$

\footnotetext{
${ }^{*}$ The second author was partially supported by the Spanish Ministerio de Ciencia e Innovación under projects MTM2011-22711.

${ }^{\dagger}$ Universität der Bundeswehr München, 85577 Neubiberg, Germany, (Thomas.Apel@unibw.de)

${ }^{\ddagger}$ Departamento de Matemáticas, E.P.I. de Gijón, Universidad de Oviedo, Campus de Gijón, 33203 Gijón, Spain (mmateos@uniovi.es).

${ }^{\S}$ Universität der Bundeswehr München, 85577 Neuibiberg, Germany, (Johannes.Pfefferer@unibw.de)

`Universtät Duisburg-Essen, Fakultät für Mathematik, Thea-Leymann-Straße 9, D-45127 Essen, Germany (Arnd.Roesch@uni-due.de)
} 


\section{DIRICHLET CONTROL IN POLYGONAL DOMAINS}

where $S u$ is the solution $y$ of the state equation

$$
-\Delta y=0 \text { in } \Omega, y=u \text { on } \Gamma,
$$

the domain $\Omega \subset \mathbb{R}^{2}$ is bounded and polygonal, $\Gamma$ is its boundary, $a<b$ and $\nu>0$ are real constants and $y_{\Omega}$ is a function whose precise regularity will be stated when necessary. Note that, for $u \in U_{a d}$, the state equation does not possess a variational solution in general, so a very weak solution is considered (see Theorem 4). We will discuss here the regularities of the optimal state $\bar{y}$, the optimal control $\bar{u}$ and the corresponding adjoint state $\bar{\varphi}$ which are limited by singularities due to corners of the domain and due to the presence of control constraints.

The classical Sobolev (Slobodetskiu) spaces are denoted by $W^{t, p}(\Omega)$ and, in the case of $p=2$, by $H^{t}(\Omega)$. As usual, for $t>0, W_{0}^{t, p}(\Omega)$ or $H_{0}^{t}(\Omega)$ will denote the closure respectively in $W^{t, p}(\Omega)$ or $H^{t}(\Omega)$ of $\mathcal{D}(\Omega)$, the space of infinitely differentiable functions with compact support in $\Omega$, and $W^{-t, q}(\Omega)$ with $q^{-1}+p^{-1}=1$ [resp. $\left.H^{-t}(\Omega)\right]$ is the dual space of $W_{0}^{t, p}(\Omega)\left[\right.$ resp. $\left.H_{0}^{t}(\Omega)\right]$.

The seminal paper on Dirichlet control problems is the work by Casas and Raymond [6]. They investigate the problem even with a semilinear state equation. Assuming a convex polygonal domain with maximal interior angle $\omega_{1}<\pi$, they prove $\bar{u} \in W^{1-1 / p, p}(\Gamma)$ and $\bar{y} \in W^{1, p}(\Omega)$ with $p<p_{\Omega}=2 /\left(2-\min \left\{\lambda_{1}, 2\right\}\right)$ and $\lambda_{1}=\pi / \omega_{1}$ both for the case with and without control constraints. Note that $p_{\Omega}>2$ due to the convexity of the domain. May, Rannacher and Vexler [14] consider the unconstrained Dirichlet control problem with linear state equation (1.1) also in convex domains and derive $\bar{u} \in H^{1-1 / p}(\Gamma)$ and $\bar{y} \in H^{3 / 2-1 / p}(\Omega)$ for $p<p_{\Omega}$. Deckelnick, Günter and Hinze [9] focus on approximation issues in the case of smooth domains (class $C^{3}$ ) in two and three space dimensions. The regularity is determined by the box constraints since corner (or edge) singularities do not occur. Finally we would like to mention the paper [15] by Of, Phan, and Steinbach where the control is searched in $H^{1 / 2}(\Omega)$ such that the state $\bar{y} \in H^{1}(\Omega)$ satisfies the weak formulation; in this case the regularity issues are less severe.

Due to the assumptions on the domain, the publications [6], 9], and [14 have in common that the adjoint problem can be solved in $H^{2}(\Omega) \cap H_{0}^{1}(\Omega)$. For that reason the very weak formulation of the state equation is well defined in these publications with test functions from $H^{2}(\Omega) \cap H_{0}^{1}(\Omega)$. This is not the case when non-convex domains are considered. Instead, the very weak fomulation of the state equation should be defined with test functions from $H_{\Delta}^{1}(\Omega) \cap H_{0}^{1}(\Omega)$, where $H_{\Delta}^{1}(\Omega):=\left\{v \in H^{1}(\Omega): \Delta v \in L^{2}(\Omega)\right\}$, see the paper by Casas, Mateos and Raymond [5, (A.16)] and also [1] for further approaches how to understand the solution of the Poisson equation with non-smooth boundary data.

In the paper at hand we prove basic regularity results for the solution of the state and adjoint state equations in Section 2, We extend in Theorem 4 to non-convex domains the well-known $H^{1 / 2}(\Omega)$ regularity of the solution of the state equation (1.1) and we prove in Theorem 7 that the maximum principle also holds for very weak solutions. Our main regularity results for the variables in the optimal control problem are proved in Section 3. Among many other results, we prove that, in the presence of control constraints and 
under minimal regularity assumptions on the data $\left(y_{\Omega} \in L^{s^{*}}(\Omega), s^{*}>2\right)$, the optimal control is continuous despite the possible non-convexity of the domain (cf. Theorem 11). The main idea is very simple: we compute explicitly the normal derivative of the singular part of the adjoint state and exploit the projection relation established by the first order optimality conditions. We also investigate the case $s^{*}=2$. This case has not been treated by the cited references for convex domains. In Section 4 we prove that, for regular data, the regularity of the optimal solution is indeed slightly better. We give conditions for the control to be in $H^{3 / 2-\varepsilon}(\Omega)$ for all $\varepsilon>0$ (Corollary 16), which is the best regularity we can expect under pointwise control constraints. These results will be helpful to derive error estimates for finite element approximations of problem $(\mathrm{P})$. The numerical analysis will be carried out in a forthcoming paper. The short Section [5] is devoted to the unconstrained case. Whereas in many other control problems the regularity of the unconstrained solution is better than that of the constrained solution, we encounter here the phenomenon that the constraint inhibits poles of the unconstrained solution. For that reason the regularity of the constrained control is determined, up to some exceptional cases (cf. Remark 12), by the largest convex angle but the regularity of the unconstrained control is determined by the overall largest angle (cf. Corollary 19).

Finally we would like to remark that sometimes the state equation is considered in the form

$$
-\Delta y=f \text { in } \Omega, y=u+g \text { on } \Gamma,
$$

but we can take the unique function $y_{0}$ which solves $-\Delta y_{0}=f$ in $\Omega, y_{0}=g$ on $\Gamma$, and replace $y:=y+y_{0}, y_{\Omega}:=y_{\Omega}-y_{0}$ and recover problem (P) with (1.1) for data sufficiently smooth.

\section{Notation and basic results for elliptic equations}

Let us denote by $M$ the number of sides of $\Gamma$ and $\left\{x_{j}\right\}_{j=1}^{M}$ its vertexes, ordered counterclockwise. For convenience denote also $x_{0}=x_{M}$ and $x_{M+1}=x_{1}$. We will denote by $\Gamma_{j}$ the side of $\Gamma$ connecting $x_{j}$ and $x_{j+1}$, and by $\omega_{j} \in(0,2 \pi)$ the angle interior to $\Omega$ at $x_{j}$, i.e., the angle defined by $\Gamma_{j}$ and $\Gamma_{j-1}$, measured counterclockwise. Notice that $\Gamma_{0}=\Gamma_{M}$. We will use $\left(r_{j}, \theta_{j}\right)$ as local polar coordinates at $x_{j}$, with $r_{j}=\left|x-x_{j}\right|$ and $\theta_{j}$ the angle defined by $\Gamma_{j}$ and the segment $\left[x_{j}, x\right]$. In order to describe the regularity of the functions near the corners, we will introduce for every $j=1, \ldots, M$ the infinite cone

$$
K_{j}=\left\{x \in \mathbb{R}^{2}: 0<r_{j}, 0<\theta_{j}<\omega_{j}\right\}
$$

and a positive number $R_{j}$ such that the sets

$$
N_{j}=\left\{x \in \mathbb{R}^{2}: 0<r_{j}<2 R_{j}, 0<\theta_{j}<\omega_{j}\right\},
$$

satisfy $N_{j} \subset \Omega$ for all $j$ and $N_{i} \cap N_{j}=\emptyset$ if $i \neq j$. 


\section{DIRICHLET CONTROL IN POLYGONAL DOMAINS}

For every $j=1, \ldots, M$ we will also consider

$$
\xi_{j}: \mathbb{R}^{2} \rightarrow[0,1]
$$

an infinitely differentiable cut-off function which is equal to 1 in the set $\left\{x \in \mathbb{R}^{2}: r_{j}<\right.$ $\left.R_{j}\right\}$ and equal to 0 in the set $\left\{x \in \mathbb{R}^{2}: r_{j}>2 R_{j}\right\}$.

We will denote by $z_{f}$ the solution of the homogeneous Dirichlet problem with distributed data

$$
-\Delta z=f \text { in } \Omega, z=0 \text { on } \Gamma \text {. }
$$

The regularity of $f$ and $z_{f}$, as well as in what sense the equation must be understood, will be stated when necessary.

For every $j=1, \ldots, M$ we will call $\lambda_{j}$ the leading singular exponent associated with the operator corresponding to the corner $x_{j}$. For the Laplace operator it is well known that $\lambda_{j}=\pi / \omega_{j}$. For convenience we will suppose that $\lambda_{1}=\min \left\{\lambda_{j}: j=1, \ldots, M\right\}$. In general, the maximum regularity in non-weighted Sobolev spaces of the solution of the Poisson problem for regular data will be given by the Sobolev exponents

$$
p_{\Omega}=\frac{2}{2-\min \left\{\lambda_{1}, 2\right\}} \text { and } t_{\Omega}=1+\lambda_{1} .
$$

This is, for $f \in C^{\infty}(\Omega)$, the solution $z_{f}$ of equation (2.1) will satisfy that $z \in W^{2, p}(\Omega) \cap$ $H^{t}(\Omega)$, for all $p<p_{\Omega}$ (cf. Grisvard [10, Th. 4.4.3.7]) and all $t<t_{\Omega}$ (cf. Dauge [7, $\S 23 . \mathrm{C}$ ]; see also Grisvard [11, Th. 2.4.3 and $\S 2.7]$ ). For less regular data $f \in W^{-1, q}(\Omega)$, the maximum regularity of the solution of the homogeneous Dirichlet problem is given by the exponent

$$
p_{D}=\frac{2}{1-\min \left\{1, \lambda_{1}\right\}} .
$$

This means that $z_{f} \in W^{1, q}(\Omega)$ if $q<p_{D}$ (cf. Dauge [8, Theorem 1.1(i)]; see also Jerison and Kenig [12, Thms. 0.5, 1.1, 1.3]). Further results on the local regularity in each corner can be stated using weighted Sobolev spaces.

We will state now two lemmas collecting several regularity results that will be used later to state the regularity of the solution of the control problem. The following lemma is a consequence of well known regularity results collected in the book by Grisvard [10]. It tells us accurately how the singularities arising from the corners behave for problems with regular data. We introduce the following sets in order to describe the singular behavior of the solution of the Poisson problem at the corners.

For $1<p<+\infty$ such that

$$
\frac{2(p-1)}{p \lambda_{j}} \notin \mathbb{Z} \forall j \in\{1, \ldots, M\}
$$

and $m \in \mathbb{Z}$, define

$$
\mathbb{J}_{p}^{m}=\left\{j \in\{1, \ldots, M\} \text { such that } 0<m \lambda_{j}<2-\frac{2}{p}\right\} .
$$




\section{DIRICHLET CONTROL IN POLYGONAL DOMAINS}

The condition in (2.2) is necessary to deduce the $W^{2, p}(\Omega)$-regularity of the regular part of the solution in the lemma below. The meaning of the sets $\mathbb{J}_{p}^{m}$ is the following:

$$
\xi_{j} r_{j}^{m \lambda_{j}} \notin W^{2, p}(\Omega) \text { for all } j \in \mathbb{J}_{p}^{m} \text {. }
$$

Notice that, for all $p<+\infty, \mathbb{J}_{p}^{m}=\emptyset$ for $m \geq 4$ since $\lambda_{j}>1 / 2$ for every possible angle and $2-2 / p<2$ for all $p<+\infty$. We also remark that $\mathbb{J}_{p}^{3} \subset \mathbb{J}_{p}^{2} \subset \mathbb{J}_{p}^{1}$.

Lemma 1. Consider $1<p<+\infty$ satisfying (2.2) and $f \in L^{p}(\Omega)$. Then there exist unique real numbers $\left(c_{j, m}\right)_{j \in \mathbb{J}_{p}^{m}}$ and a unique solution $z_{f} \in H^{1}(\Omega)$ of problem (2.1) such that

$$
z_{f}=z_{r e g}+\sum_{m=1}^{3} \sum_{j \in J_{p}^{m}} c_{j, m} \xi_{j} r_{j}^{m \lambda_{j}} \sin \left(m \lambda_{j} \theta_{j}\right)
$$

where $z_{\text {reg }} \in W^{2, p}(\Omega)$ and the $\xi_{j}$ are the cut-off functions introduced above.

Proof. The result is a direct consequence of [10, Theorem 4.4.3.7]. This result can be applied since $z_{f} \in H^{1}(\Omega)$ thanks to [10, Lemma 4.4.3.1].

Corollary 2. If $f \in L^{2}(\Omega)$, then $z_{f} \in H^{t}(\Omega)$ for all $t<1+\lambda_{1}$ and $\partial_{n} z_{f} \in L^{2}(\Gamma)$.

Proof. To prove this fact, we apply Lemma 1; the regular part is in $H^{2}(\Omega)$; on the other hand, since $\lambda_{j} \geq \lambda_{1}>1 / 2$ and $m \geq 1$, then $\xi_{j} r_{j}^{m \lambda_{j}} \in H^{t}(\Omega)$ for all $t<1+\lambda_{1}$. From this we obtain that $z_{f} \in H^{t}(\Omega)$. Since we can choose $t>3 / 2$ we have $\partial_{n} z_{f} \in L^{2}(\Gamma)$.

The next result states the regularity of the solution of problems with boundary data in $W^{1-1 / p^{*}, p^{*}}(\Gamma)$ with $p^{*} \geq 2$. Let us recall (cf. [10, Theorem 1.5.2.3]) that the trace of any function $z \in W^{1, p^{*}}(\Omega)$ is in $W^{1-1 / p^{*}, p^{*}}(\Gamma)$, the trace mapping is onto, and, for $p^{*}>2$, this space can be characterized as

$$
W^{1-1 / p^{*}, p^{*}}(\Gamma)=\left\{g \in \prod_{i=1}^{M} W^{1-1 / p^{*}, p^{*}}\left(\Gamma_{i}\right): g \text { is continuous at every corner } x_{j}\right\} .
$$

For $p^{*}=2$ the continuity requirement in the corners can be weakened to an integral condition, see [10, Theorem 1.5.2.3(c)].

Lemma 3. Let $g \in W^{1-1 / p^{*}, p^{*}}(\Gamma)$ for some $p^{*} \geq 2$. Then there exists a unique solution $z \in W^{1, p}(\Omega)$, for all $p \leq p^{*}, p<p_{D}$, of the equation

$$
-\Delta z=0 \text { in } \Omega, z=g \text { on } \Gamma \text {. }
$$

Proof. Due to the trace theorem, there exists a function $G \in W^{1, p^{*}}(\Omega)$ such that its trace is $\gamma G=g$. Moreover, we have that $\Delta G \in W^{-1, p^{*}}(\Omega)$. If we define $\zeta=z-G$, then it satisfies the boundary value problem

$$
-\Delta \zeta=-\Delta G \text { in } \Omega, \zeta=0 \text { on } \Gamma .
$$




\section{DIRICHLET CONTROL IN POLYGONAL DOMAINS}

Let $p \leq p^{*}, p<p_{D}$. Then $\zeta \in W_{0}^{1, p}(\Omega)$ (see above or cf. Dauge [8, Theorem 1.1(i)]; see also Jerison and Kenig [12, Thms. 0.5, 1.1, 1.3]) and hence so does $z \in W^{1, p}(\Omega)$.

Since the space of controls is $L^{2}(\Gamma)$, the state equation must be understood in the transposition sense. Following [2, 5], for $u \in L^{2}(\Gamma)$, we will say that $y \in L^{2}(\Omega)$ is a solution of (1.1) if for every $f \in L^{2}(\Omega)$

$$
\int_{\Omega} y f d x=-\int_{\Gamma} u \partial_{n} z_{f} d \sigma(x)
$$

where $z_{f}$ is defined in Lemma 1, The definition makes sense thanks to Corollary 2 ,

Existence, uniqueness and regularity of the solution in $y \in H^{1 / 2}(\Omega)$ if $\Omega$ is convex domains is a classical result and can be proved via transposition and interpolation. Let us briefly recall how this result is obtained for a convex domain. Consider the solution operator $S$ of (1.1) with $S u=y$. Due to the Lemma 3 it is

$$
S \in \mathcal{L}\left(H^{1 / 2}(\Gamma), H^{1}(\Omega)\right) .
$$

Using the classical transposition method (cf. [13]) we also have that

$$
S \in \mathcal{L}\left(H^{-1 / 2}(\Gamma), L^{2}(\Omega)\right) .
$$

The final result is obtained by interpolation using that

$$
L^{2}(\Gamma)=\left[H^{1 / 2}(\Gamma), H^{-1 / 2}(\Gamma)\right]_{1 / 2} \text { and } H^{1 / 2}(\Omega)=\left[H^{1}(\Omega), L^{2}(\Omega)\right]_{1 / 2},
$$

see e.g. [13, Chap. 1, Eq. (2.41)]) for the first result and notice that interpolation results are valid for spaces posed on Lipschitz domains (cf. [3, Theorem 12.2.7] or [12]). We cannot use this scheme straightforward because (2.6) uses explicitly that for every $f \in L^{2}(\Omega), z_{f} \in H^{2}(\Omega)$, and this is not true for non-convex polygonal domains.

For problems posed on non-convex polygonal domains, Berggren [2, Theorem 4.2] proves existence, uniqueness and regularity of the solution $y \in H^{t}(\Omega)$ for every $0<t<$ $\epsilon \leq 1 / 2$ ( $\epsilon$ depends on the domain). We can also achieve $y \in H^{1 / 2}(\Omega)$ in non-convex domains. The proof of our following result uses interpolation spaces; a different proof by using integral operators is given in [1].

Theorem 4. For every $u \in L^{2}(\Gamma)$ there exists a unique solution $y \in H^{1 / 2}(\Omega)$ of (1.1) and

$$
\|y\|_{H^{1 / 2}(\Omega)} \leq C\|u\|_{L^{2}(\Gamma)} .
$$

Proof. Notice that for $0<\varepsilon<1 / 2$, we also have that, for $\theta=1 /(1+2 \varepsilon)$,

$$
L^{2}(\Gamma)=\left[H^{1 / 2}(\Gamma), H^{-\varepsilon}(\Gamma)\right]_{\theta} \text { and } H^{1 / 2}(\Omega)=\left[H^{1}(\Omega), H^{1 / 2-\varepsilon}(\Omega)\right]_{\theta}
$$

and therefore the result will be true if we can prove that $S \in \mathcal{L}\left(H^{-\varepsilon}(\Gamma), H^{1 / 2-\varepsilon}(\Omega)\right)$ for some $\varepsilon>0$. 


\section{DIRICHLET CONTROL IN POLYGONAL DOMAINS}

Fix $0<\varepsilon<\min \left\{\lambda_{1}-1 / 2,1 / 2\right\}$. For any $u \in H^{-\varepsilon}(\Gamma)$, we will say that $y=S u$ if for every $f \in L^{2}(\Omega)$

$$
\int_{\Omega} y f d x=-\left\langle u, \partial_{n} z_{f}\right\rangle_{H^{-\varepsilon}(\Gamma), H^{\varepsilon}(\Gamma)} .
$$

Notice that since $\varepsilon<\lambda_{1}-1 / 2$ we have that $z_{f} \in H^{3 / 2+\varepsilon}(\Omega)$ and that $\partial_{n} z_{f} \in \prod_{j=1}^{M} H^{\varepsilon}\left(\Gamma_{j}\right)=$ $H^{\varepsilon}(\Gamma)$ because $\varepsilon<1 / 2$ (cf. [10, Theorem 1.5.2.3(a)]). Moreover, due to the trace theorem and elliptic regularity, we have that

$$
\left\|\partial_{n} z_{f}\right\|_{H^{\varepsilon}(\Gamma)} \leq C\|f\|_{H^{\varepsilon-1 / 2}(\Omega)} .
$$

Notice also that if $u \in H^{1 / 2}(\Gamma)$ then the unique variational solution $y \in H^{1}(\Omega)$ of

$$
-\Delta y=0 \text { in } \Omega, y=u \text { on } \Gamma
$$

is a solution of (2.7) and if $u \in L^{2}(\Gamma)$, then (2.7) is the same as (2.4).

Let us prove uniqueness of the solution of (2.7) in $L^{2}(\Omega)$ first. If $u=0$ and $y=S u \in$ $L^{2}(\Omega)$, then, taking $f=y$ as test function in (2.7) we get $\int_{\Omega} y^{2} d x=0$, and therefore $y \equiv 0$. Since the problem is linear, the solution is unique.

We next prove existence of a solution $y \in H^{1 / 2-\varepsilon}(\Omega)$ of (2.7). We know (cf. [10, Theorem 1.4.2.4]) that $H^{1 / 2-\varepsilon}(\Omega)=H_{0}^{1 / 2-\varepsilon}(\Omega)$ and hence $\left(H^{1 / 2-\varepsilon}(\Omega)\right)^{\prime}=H^{\varepsilon-1 / 2}(\Omega)$. Denote

$$
\mathcal{F}=\left\{f \in L^{2}(\Omega) \text { such that }\|f\|_{H^{\varepsilon-1 / 2}(\Omega)}=1\right\} .
$$

For any $u \in H^{1 / 2}(\Gamma), y=S u$, we use that $L^{2}(\Omega)$ is dense in $H^{\varepsilon-1 / 2}(\Omega)$ and (2.8) to obtain

$$
\begin{aligned}
\|y\|_{H^{1 / 2-\varepsilon}(\Omega)} & =\sup _{f \in \mathcal{F}}\langle f, y\rangle_{H^{\varepsilon-1 / 2}(\Omega), H^{1 / 2-\varepsilon}(\Omega)}=\sup _{f \in \mathcal{F}} \int_{\Omega} y f d x \\
& =\sup _{f \in \mathcal{F}}-\left\langle u, \partial_{n} z_{f}\right\rangle_{H^{-\varepsilon}(\Gamma), H^{\varepsilon}(\Gamma)} \leq \sup _{f \in \mathcal{F}}\left\|\partial_{n} z_{f}\right\|_{H^{\varepsilon}(\Gamma)}\|u\|_{H^{-\varepsilon}(\Gamma)} \\
& \leq C \sup _{f \in \mathcal{F}}\|f\|_{H^{\varepsilon-1 / 2}(\Omega)}\|u\|_{H^{-\varepsilon}(\Gamma)}=C\|u\|_{H^{-\varepsilon}(\Gamma)} .
\end{aligned}
$$

Take a sequence $u_{k} \in H^{1 / 2}(\Gamma), u_{k} \rightarrow u \in H^{-\varepsilon}(\Gamma)$, and let $y_{k}=S u_{k}$. We have just proved that

$$
\left\|y_{k}-y_{m}\right\|_{H^{1 / 2-\varepsilon}(\Omega)} \leq\left\|u_{k}-u_{m}\right\|_{H^{-\varepsilon}(\Gamma)}
$$

and therefore $y_{k}$ converges in $H^{1 / 2-\varepsilon}(\Omega)$ to some $y \in H^{1 / 2-\varepsilon}(\Omega)$ that is (the unique) solution of the equation:

$$
\begin{aligned}
\int_{\Omega} y f d x & =\int_{\Omega} \lim _{k} y_{k} f d x=\lim _{k} \int_{\Omega} y_{k} f d x \\
& =\lim _{k}-\left\langle u_{k}, \partial_{n} z_{f}\right\rangle_{H^{-\varepsilon}(\Gamma), H^{\varepsilon}(\Gamma)}=-\left\langle\lim _{k} u_{k}, \partial_{n} z_{f}\right\rangle_{H^{-\varepsilon}(\Gamma), H^{\varepsilon}(\Gamma)} \\
& =-\left\langle u, \partial_{n} z_{f}\right\rangle_{H^{-\varepsilon}(\Gamma), H^{\varepsilon}(\Gamma)} .
\end{aligned}
$$

Finally, (2.9) implies that $S \in \mathcal{L}\left(H^{-\varepsilon}(\Gamma), H^{1 / 2-\varepsilon}(\Omega)\right)$ and the proof is complete. 


\section{DIRICHLET CONTROL IN POLYGONAL DOMAINS}

The next result is rather technical. It will be used to describe precisely the structure of the optimal state in the proof of Theorem 13 below. In that result we will be able to write the control as the sum of a regular part and a singular part. We show in Lemma 2.5 how to solve the state equation for singular boundary data. Besides the usual regular and singular parts that we described in Lemma 1, a new singular part of the solution arises from the boundary data.

Define the jump functions at the corners

$$
\chi_{j}=\left\{\begin{aligned}
1 & \text { on }\left\{x \in \partial K_{j}: \theta_{j}=0\right\} \\
-1 & \text { on }\left\{x \in \partial K_{j}: \theta_{j}=\omega_{j}\right\}
\end{aligned}\right.
$$

Notice that $\chi_{j}=1$ on $\Gamma_{j}$ and $\chi_{j}=-1$ on $\Gamma_{j-1}$.

Lemma 5. Consider any pair of subsets $\mathbb{H}^{1}, \mathbb{H}^{2} \subset\{1, \ldots, M\}$ and real numbers $-1 / 2<$ $\eta_{j, 1}$ and $a_{j, 1}$ for all $j \in \mathbb{H}^{1}$ and $0<\eta_{j, 2}$ and $a_{j, 2}$ for all $j \in \mathbb{H}^{2}$ such that $\eta_{j, n} / \lambda_{j} \notin \mathbb{Z}$ for any $j \in \mathbb{H}^{n}, n=1,2$. Define

$$
u=\sum_{j \in \mathbb{H}^{1}} a_{j, 1} \xi_{j} r_{j}^{\eta_{j, 1}}+\sum_{j \in \mathbb{H}^{2}} \chi_{j} a_{j, 2} \xi_{j} r_{j}^{\eta_{j, 2}} \text { on } \Gamma
$$

take $p$ such that

$$
1<p<\inf \left\{\frac{2}{1-\eta_{j, n}}: j \in \mathbb{H}^{n}, n=1,2 \text { and } \eta_{j, n}<1\right\}
$$

where we consider inf $\emptyset=+\infty$ and define $\mathbb{J}_{p}^{m}$ as in (2.3). Then there exist unique real numbers $\left(c_{j, m}\right)_{j \in \mathbb{J}_{p}^{m}}, m=1,2,3$ and a unique solution $y \in H^{1 / 2}(\Omega)$ of equation (1.1) such that

$$
y=y_{r e g}+\sum_{n=1}^{2} \sum_{j \in \mathbb{H}^{n}} a_{j, n} \xi_{j} r_{j}^{\eta_{j, n}} s_{j, n}\left(\theta_{j}\right)+\sum_{m=1}^{3} \sum_{j \in \mathbb{J}_{p}^{m}} c_{j, m} \xi_{j} r_{j}^{m \lambda_{j}} \sin \left(m \lambda_{j} \theta_{j}\right),
$$

where

$$
s_{j, n}\left(\theta_{j}\right)=\frac{(-1)^{n+1}-\cos \left(\eta_{j} \omega_{j}\right)}{\sin \left(\eta_{j} \omega_{j}\right)} \sin \left(\eta_{j} \theta_{j}\right)+\cos \left(\eta_{j} \theta_{j}\right)
$$

and $y_{\text {reg }} \in W^{2, p}(\Omega)$. If, further, $\eta_{j, 1}>0$ for all $j \in \mathbb{H}^{1}$, then $y \in H^{1}(\Omega)$.

Proof. Since $\eta_{j, 1}>-1 / 2$, we have that $u \in L^{2}(\Gamma)$ and $y \in H^{1 / 2}(\Omega)$ thanks to Theorem 4. If also $\eta_{j, 1}>0$, then $u \in H^{1 / 2}(\Gamma)$ and hence Lemma 3 gives us that $y \in H^{1}(\Omega)$.

Notice next that $\eta_{j, n}>-1 / 2$ implies that $2 /\left(1-\eta_{j, n}\right)>4 / 3$ and $p$ is well defined.

A direct computation shows that for $n=1,2, y_{j, n}=r_{j}^{\eta_{j, n}} s_{j, n}(\theta)$ are respectively the solutions of the problems

$$
-\Delta y_{j, 1}=0 \text { in } K_{j}, y_{j, 1}=r_{j}^{\eta_{j, 1}} \text { on } \partial K_{j},
$$




$$
-\Delta y_{j, 2}=0 \text { in } K_{j}, y_{j, 2}=\chi_{j} r_{j}^{\eta_{j, 2}} \text { on } \partial K_{j} .
$$

Since $\Delta y_{j, 1}=0$, we have that

$$
\Delta \sum_{n=1}^{2} \sum_{j \in \mathbb{J}^{n}} \xi_{j} y_{j, n}=\sum_{j=1}^{n} \sum_{j \in \mathbb{J}^{n}}\left(y_{j, n} \Delta \xi_{j}+2 \nabla y_{j, n} \nabla \xi_{j}\right) .
$$

Since $\left|\nabla y_{j, n}\right| \leq C r_{j}^{\eta_{j, n}-1}$, the condition imposed on $p$ implies that

$$
f=\Delta \sum_{n=1}^{2} \sum_{j \in \mathbb{J}^{n}} a_{j, n} \xi_{j} y_{j, n} \in L^{p}(\Omega),
$$

and we can write $y=y_{f}+\sum_{n=1}^{2} \sum_{j \in \mathbb{H}^{n}} a_{j, n} \xi_{j} y_{j, n}$, where

$$
-\Delta y_{f}=f \text { in } \Omega, y_{f}=0 \text { on } \Gamma \text {. }
$$

Applying Lemma 1 we obtain that $y_{f}=y_{r e g}+\sum_{m=1}^{3} \sum_{j \in \mathbb{J}_{p}^{m}} c_{j, m} \xi_{j} r_{j}^{m \lambda_{j}} \sin \left(m \lambda_{j} \theta_{j}\right)$ and the proof is complete.

Although the maximum principle is a well known result for weak solutions of equation (1.1) (see the celebrated paper by Stampacchia [16]), we have not been able to find a reference of its validity for solutions defined in the transposition sense (2.4). For the sake of completeness, we include such a result. First, we prove the following technical lemma.

Lemma 6. Consider $f \in L^{2}(\Omega), f \geq 0$ and let $z_{f} \in H_{0}^{1}(\Omega)$ be the solution of equation (2.1). Then $\partial_{n} z_{f} \leq 0$ a.e. on $\Gamma$.

Proof. Take $u \in C^{\infty}(\Gamma), u \geq 0$. Thanks to Lemma 3, the solution of equation (1.1) satisfies $y \in H^{1}(\Omega)$ and the maximum principle for weak solutions, as proved by Stampacchia [16], holds. Therefore $y \geq 0$. Integration by parts shows then that

$$
0 \leq \int_{\Omega} y f d x=-\int_{\Gamma} u \partial_{n} z_{f} d \sigma(x)
$$

and the result follows by the usual density argument.

Theorem 7. Consider $u \in L^{\infty}(\Gamma)$. Then the solution $y$ of equation (1.1) belongs to $L^{\infty}(\Omega)$ and

$$
\|y\|_{L^{\infty}(\Omega)} \leq\|u\|_{L^{\infty}(\Gamma)} .
$$

Proof. Define $K=\|u\|_{L^{\infty}(\Gamma)}$. We will prove that $y \leq K$ a.e. on $\Omega$, the proof for $-y \leq K$ being analogous. 
We already know that $y \in H^{1 / 2}(\Omega) \hookrightarrow L^{2}(\Omega)$ (cf. Theorem 4). Define $y_{K}=y-K$ and $y_{K}^{+}=\max \left(y_{K}, 0\right) \in L^{2}(\Omega)$. For every $f \in L^{2}(\Omega)$ we deduce from (2.1) that

$$
\int_{\Omega} y_{K} f d x=-\int_{\Gamma}(u-K) \partial_{n} z_{f} d \sigma(x)
$$

We take $f=y_{K}^{+} \geq 0$. For this choice of $f$, we know from Lemma 6 that $\partial_{n} z_{f} \leq 0$ a.e. on $\Gamma$ and therefore

$$
0 \leq \int_{\Omega}\left(y_{K}^{+}\right)^{2} d x=\int_{\Omega} y_{K} y_{K}^{+} d x=-\int_{\Gamma}(u-K) \partial_{n} z_{f} d \sigma(x) \leq 0 .
$$

So $y_{K}^{+} \equiv 0$ and $y \leq K$.

\section{Main regularity results for the control problem}

The following result is standard and the proof can be found in [6]. Though in that reference only convex domains are taken into account, this result is independent of the convexity of the domain, once we have proved Corollary 2 and Theorem 4. Here and in the rest of the paper, $\operatorname{Proj}_{[a, b]}(c)=\min \{b, \max \{a, c\}\}$ for any real numbers $a, b, c$.

Lemma 8. Suppose $y_{\Omega} \in L^{2}(\Omega)$. Then problem $(P)$ has a unique solution $\bar{u} \in L^{2}(\Gamma)$ with related state $\bar{y} \in H^{1 / 2}(\Omega)$ and adjoint state $\bar{\varphi} \in H_{0}^{1}(\Omega)$. The following optimality system is satisfied:

$$
\begin{aligned}
\bar{u}(x) & =\operatorname{Proj}_{[a, b]}\left(\frac{1}{\nu} \partial_{n} \bar{\varphi}(x)\right) \text { for a.e. } x \in \Gamma, \\
-\Delta \bar{y} & =0 \text { in } \Omega, \bar{y}=\bar{u} \text { on } \Gamma \\
-\Delta \bar{\varphi} & =\bar{y}-y_{\Omega} \text { in } \Omega, \bar{\varphi}=0 \text { on } \Gamma .
\end{aligned}
$$

As in the proof of Theorem 4, the solution of the state equation (3.2) must be understood in the transposition sense, whereas the adjoint state equation (3.3) has a variational solution.

Next we state a regularity result for the adjoint state in the framework of classical Sobolev-Slobodetskil spaces. In the rest of this section we will suppose that $y_{\Omega} \in L^{s^{*}}(\Omega)$ where, $2 \leq s^{*}<+\infty$ satisfies

$$
\frac{2\left(s^{*}-1\right)}{\lambda_{j} s^{*}} \notin \mathbb{Z} \forall j \in\{1, \ldots, M\} .
$$

Theorem 9. There exist a unique function $\bar{\varphi}_{\text {reg }} \in W^{2, s^{*}}(\Omega)$ and unique real numbers $\left(\hat{c}_{j, m}\right)_{j \in \mathbb{J}_{s^{*}}^{m}}$, where $\mathbb{J}_{s^{*}}^{m}$ is defined in (2.3), such that

$$
\bar{\varphi}=\bar{\varphi}_{r e g}+\sum_{m=1}^{3} \sum_{j \in J_{s^{*}}^{m}} \hat{c}_{j, m} \xi_{j} r_{j}^{m \lambda_{j}} \sin \left(m \lambda_{j} \theta_{j}\right) .
$$




\section{DIRICHLET CONTROL IN POLYGONAL DOMAINS}

Proof. Since the problem is control constrained, $\bar{u} \in L^{\infty}(\Gamma)$, and by the maximum principle proved in Theorem [7, $\bar{y} \in L^{\infty}(\Omega)$. Therefore, $\bar{y}-y_{\Omega} \in L^{s^{*}}(\Omega)$, and we can use Lemma 1; since the related adjoint state $\bar{\varphi}$ is the solution of (3.3) we have that there exist unique $\bar{\varphi}_{r e g} \in W^{2, s^{*}}(\Omega)$ and $\left(\hat{c}_{j, m}\right)_{j \in \mathbb{J}_{s}^{m}}$ such that relation (3.5) holds.

For any $s \geq 2$ we define the set

$$
\mathbb{H}_{s}^{1}=\left\{j \in \mathbb{J}_{s}^{1}: \lambda_{j}>1\right\}
$$

and for $s \geq 2$ and $m=2,3$

$$
\mathbb{H}_{s}^{m}=\left\{j \in \mathbb{J}_{s}^{m}: \hat{c}_{j, 1}=0\right\},
$$

where the coefficients $\hat{c}_{j, m}$ are the coefficients obtained in Theorem 9, Notice that the indexes in $\mathbb{H}_{s}^{1}$ correspond to convex corners. The indexes in $\mathbb{H}_{s}^{m}$ correspond to those non-convex corners where the main part of the singularity of the adjoint state vanishes, and hence the behavior of the solution at those non-convex corners can be somehow compared to the behavior of the solution at the convex corners. Notice also that

$$
\cup_{m=1}^{3} \mathbb{J}_{s}^{m}=\cup_{m=1}^{3} \mathbb{H}_{s}^{m} \bigcup\left\{j \in \mathbb{J}_{s}^{1}: \lambda_{j}<1 \text { and } \hat{c}_{j, 1} \neq 0\right\}
$$

Consider also $p \geq 2$ such that for $m=1,2,3$

$$
p \leq s^{*}, p<\frac{2}{2-m \lambda_{j}} \text { if } j \in \mathbb{H}_{s^{*}}^{m} .
$$

This condition on $p$ appears in a natural way in the proof of Theorem 11, see (3.10). If $s^{*}>2$, then we can choose $p>2$. With this choice we have that

Lemma 10. Let $p$ satisfy (3.6) and for $m=1,2,3$ consider $j \in \mathbb{H}_{s^{*}}^{m}$. Then

$$
\xi_{j} r_{j}^{m \lambda_{j}-1} \in W^{1-1 / p, p}(\Gamma) .
$$

Proof. Take $j \in \cup_{m=1}^{3} \mathbb{H}_{s^{*}}^{m}$ and consider $N_{j}$ the bounded cone of radius $2 R_{j}$ defined in Section 2. We first prove that $u_{j}=r_{j}^{m \lambda_{j}-1} \in W^{1, p}\left(N_{j}\right)$.

Since $j \in \cup_{m=1}^{3} \mathbb{H}_{s^{*}}^{m}$, then $m \lambda_{j}>1$, so $m \lambda_{j}-1>0$ and $r_{j}^{m \lambda_{j}-1} \in C\left(\bar{N}_{j}\right) \subset L^{p}\left(N_{j}\right)$. On the other hand $\left|\nabla u_{j}\right|=\left(m \lambda_{j}-1\right) r_{j}^{m \lambda_{j}-2}$, and making the usual change of variables to polar coordinates, we have

$$
\iint_{N_{j}}\left|\nabla u_{j}\right|^{p} d x=\left(m \lambda_{j}-1\right)^{p} \omega_{j} \int_{0}^{2 R_{j}} r_{j} r_{j}^{\left(m \lambda_{j}-2\right) p} d r
$$

the last integral being convergent if and only if $\left(m \lambda_{j}-2\right) p+1>-1$. Taking into account that $j \in \mathbb{J}_{s^{*}}^{m}$ implies $m \lambda_{j}<2$, the previous condition is fulfilled if and only if $p<\frac{2}{2-m \lambda_{j}}$, which is the assumption.

Relation (3.7) now follows from the smoothness of the cut-off function, the trace theorem and the continuity of $\xi_{j} r_{j}^{m \lambda_{j}-1}$. 


\section{DIRICHLET CONTROL IN POLYGONAL DOMAINS}

Theorem 11. Let $p$ satisfy (3.6). Then, the optimal control $\bar{u}$ belongs to $W^{1-1 / p, p}(\Gamma)$, the optimal state $\bar{y}$ belongs to $W^{1, q}(\Omega)$ for all $q \leq p, q<p_{D}$. In particular, if $s^{*}>2$, both are continuous functions.

Proof. We will exploit the projection relation (3.1) and the expression for the adjoint state obtained in (3.5).

Notice first that $\bar{\varphi}_{r e g} \in W^{2, s^{*}}(\Omega)$ and $\bar{\varphi}_{r e g}=0$ on $\Gamma$, so $\partial_{n} \bar{\varphi}_{r e g} \in W^{1-1 / s^{*}, s^{*}}(\Gamma)$ (cf. [5, Lemma A.2] for the case $s^{*}=2$ or [4 for the case $s^{*}>2$ ). Moreover, if $s^{*}>2$, then $\partial_{n} \bar{\varphi}_{\text {reg }}\left(x_{j}\right)=0$ on every corner the normal derivative of the regular part is a continuous function on $\Gamma$.

We are going to compute now the normal derivative of the singular part. For any $m \in\{1,2,3\}$ and $j \in \mathbb{J}_{s^{*}}^{m}$, we have $\partial_{n} \xi_{j} r_{j}^{m \lambda_{j}} \sin \left(m \lambda_{j} \theta_{j}\right) \in C^{\infty}\left(\Gamma \backslash\left\{x_{j}\right\}\right)$, so we have that for every compact set $\mathcal{K} \subset \Gamma \backslash\left\{x_{j}: j \in \mathbb{J}_{s^{*}}^{1}\right\}$

$$
\partial_{n} \bar{\varphi} \in W^{1-1 / s^{*}, s^{*}}(\mathcal{K}) .
$$

Near the corners, for $r_{j}<R_{j}$ we have on $\Gamma_{j}$ (where $\theta_{j}=0$ ) that

$$
\begin{aligned}
\partial_{n} r_{j}^{m \lambda_{j}} \sin \left(m \lambda_{j} \theta_{j}\right) \xi_{j} & =-\frac{1}{r_{j}} \partial_{\theta} r_{j}^{m \lambda_{j}} \sin \left(m \lambda_{j} \theta_{j}\right) \\
& =-m \lambda_{j} r_{j}^{m \lambda_{j}-1} \cos \left(m \lambda_{j} 0\right)=-m \lambda_{j} r_{j}^{m \lambda_{j}-1}
\end{aligned}
$$

and on $\Gamma_{j-1}\left(\right.$ where $\left.\theta_{j}=\omega_{j}\right)$ that

$$
\begin{aligned}
\partial_{n} r_{j}^{m \lambda_{j}} \sin \left(m \lambda_{j} \theta_{j}\right) \xi_{j} & =\frac{1}{r_{j}} \partial_{\theta} r_{j}^{m \lambda_{j}} \sin \left(m \lambda_{j} \theta_{j}\right) \\
& =m \lambda_{j} r_{j}^{m \lambda_{j}-1} \cos \left(m \frac{\pi}{\omega_{j}} \omega_{j}\right)=(-1)^{m} m \lambda_{j} r_{j}^{m \lambda_{j}-1} .
\end{aligned}
$$

Next we will distinguish two cases.

Case 1: if $j \in \cup_{m=1}^{3} \mathbb{H}_{s^{*}}^{m}$ then $m \lambda_{j}>1$ and hence the limit of both expressions is zero as $r_{j} \rightarrow 0$. Noticing (3.7), we have that the choice of the exponent $p$ made in (3.6) gives us

$$
\partial_{n}\left(\bar{\varphi}_{r e g}+\sum_{m=1}^{3} \sum_{j \in \mathbb{H}_{s^{*}}^{m}} \hat{c}_{j, m} \xi_{j} r_{j}^{m \lambda_{j}} \sin \left(m \lambda_{j} \theta_{j}\right)\right) \in W^{1-1 / p, p}(\Gamma) .
$$

So far, we can deduce that, for every compact set $\mathcal{K} \subset \Gamma \backslash\left\{x_{j}: \lambda_{j}<1, \hat{c}_{j, 1} \neq 0\right\}$

$$
\partial_{n} \bar{\varphi} \in W^{1-1 / p, p}(\mathcal{K}) .
$$

Case 2: Now $j \in \mathbb{J}_{s^{*}}^{1}, \lambda_{j}<1$, and $\hat{c}_{j, 1} \neq 0$. We have $(-1)^{m}=-1$ and $m \lambda_{j}-1<0$, therefore using expressions (3.8) and (3.9) we have

$$
\lim _{x \rightarrow x_{j}} \partial_{n} \hat{c}_{j, 1} r_{j}^{\lambda_{j}} \sin \left(\lambda_{j} \theta_{j}\right) \xi_{j}=-\operatorname{sign}\left(\hat{c}_{j, 1}\right) \infty .
$$




\section{DIRICHLET CONTROL IN POLYGONAL DOMAINS}

If it happens that also $j \in \mathbb{J}_{s^{*}}^{2} \cup \mathbb{J}_{s^{*}}^{3}$, we have that for $m=2$ and $m=3, m \lambda_{j}-1>0$ and again the limit of both (3.8) and (3.9) is zero. So we have that

$$
\lim _{x \rightarrow x_{j}} \partial_{n} \sum_{m=1}^{3} \hat{c}_{j, m} r_{j}^{m \lambda_{j}} \sin \left(m \lambda_{j} \theta_{j}\right) \xi_{j}=-\operatorname{sign}\left(\hat{c}_{j, 1}\right) \infty .
$$

If $s^{*}>2$, also on this corner

$$
\lim _{x \rightarrow x_{j}} \partial_{n} \bar{\varphi}_{r e g}(x)=0,
$$

and, trivially

$$
\begin{aligned}
\lim _{x \rightarrow x_{j}} \partial_{n} \bar{\varphi}(x) & =\lim _{x \rightarrow x_{j}}\left(\partial_{n} \bar{\varphi}_{r e g}(x)+\partial_{n} \sum_{m=1}^{3} \hat{c}_{j, m} r_{j}^{m \lambda_{j}} \sin \left(m \lambda_{j} \theta_{j}\right) \xi_{j}\right) \\
& =-\operatorname{sign}\left(\hat{c}_{j, 1}\right) \infty .
\end{aligned}
$$

If $s^{*}=2$, as we said at the beginning of the proof, $\partial_{n} \bar{\varphi}_{r e g} \in H^{1 / 2}(\Gamma)$, so it needs not be even a bounded function. Nevertheless, since the singular part behaves like a negative power of $r_{j}$, this term dominates and we also have that (3.12) holds.

As a consequence, there exists $\rho_{j}>0$ such that for $x \in \Gamma$ with $\left|x-x_{j}\right|<\rho_{j}$ either $\operatorname{Proj}_{[a, b]} \partial_{n} \bar{\varphi} \equiv a$ or $\operatorname{Proj}_{[a, b]} \partial_{n} \bar{\varphi} \equiv b$ depending on the sign of $\hat{c}_{j, 1}$. So the control is flat near non-convex corners. This, together with the projection formula (3.1) and (3.11) implies that the optimal control belongs to $W^{1-1 / p, p}(\Gamma)$. Finally, the regularity of the optimal state $\bar{y}$ follows from Lemma 3 ,

Remark 12. We would like to remark that the case of having $\hat{c}_{j, 1}=0$ can be seen as a "rare" case in practice (although this can happen; see Example 14 below). So the "normal" case is that $\mathbb{H}_{s}^{m}=\emptyset$ for $m=2,3$. In this case, in the choice of $p$ made in (3.6) the indexes $m=2,3$ are excluded, and hence $p$ will only depend $\max \left\{\omega_{j}: \omega_{j}<\pi\right\}$, so we get the same regularity for the control as that obtained in [6] for convex domains.

To describe more accurately the regularity of the state and the control, we have to introduce some further notation. Consider the coefficients $\hat{c}_{j, m}$ obtained in Theorem 9 and define the coefficients

$$
a_{j, m}=\left\{\begin{array}{cc}
\frac{-m \lambda_{j} \hat{c}_{j, m}}{\nu} & \text { if } 0 \in[a, b] \\
0 & \text { if } 0 \notin[a, b]
\end{array}\right.
$$

and the functions

$$
s_{j, m}\left(\theta_{j}\right)=\frac{(-1)^{m+1}-\cos \left(\left(m \lambda_{j}-1\right) \omega_{j}\right)}{\sin \left(\left(m \lambda_{j}-1\right) \omega_{j}\right)} \sin \left(\left(m \lambda_{j}-1\right) \theta_{j}\right)+\cos \left(\left(m \lambda_{j}-1\right) \theta_{j}\right) .
$$

The sets $\mathbb{H}_{s}^{m}$ will be used now to describe the singular part of the state and the control: if $j \in \mathbb{H}_{s}^{m}$, then $\xi_{j} r_{j}^{m \lambda_{j}-1} \notin W^{1, s}(\Omega)$ and $\xi_{j} r_{j}^{m \lambda_{j}-1} \notin W^{1-1 / s, s}(\Gamma)$. Regarding (3.16) , we also mention that if $\lambda_{j} \leq 1-2 / s$, then $\xi_{j} r_{j}^{\lambda_{j}} \notin W^{1, s}(\Omega)$. 
Theorem 13. Assume further that $a b \neq 0$. Then there exist a unique $\bar{u}_{r e g} \in W^{1-1 / s^{*}, s^{*}}(\Gamma)$, a unique $\bar{y}_{\text {reg }} \in W^{1, s}(\Omega)$, for all $s \leq s^{*}, s<p_{D}$, and unique real numbers $\left(c_{j}\right)_{\lambda_{j} \leq 1-2 / s}$ such that

$$
\begin{array}{r}
\bar{u}(x)=\bar{u}_{r e g}+\sum_{m=1,3} \sum_{j \in \mathbb{H}_{s^{*}}^{m}} a_{j, m} \xi_{j} r^{m \lambda_{j}-1}+\sum_{j \in \mathbb{H}_{s^{*}}^{2}} \chi_{j} a_{j, 2} \xi_{j} r^{2 \lambda_{j}-1} \\
\bar{y}=\bar{y}_{r e g}+\sum_{m=1}^{3} \sum_{j \in \mathbb{H}_{s}^{m}} a_{j, m} \xi_{j} r_{j}^{m \lambda_{j}-1} s_{j, m}\left(\theta_{j}\right)+\sum_{\lambda_{j} \leq 1-2 / s} c_{j} \xi_{j} r_{j}^{\lambda_{j}} \sin \left(\lambda_{j} \theta_{j}\right)
\end{array}
$$

where the $\chi_{j}$ are the jump functions at the corners defined in (2.10), the $s_{j, m}(\theta)$ are defined in (3.14) and the $\xi_{j}$ are the cut-off functions.

Proof. From the considerations in the proof of Theorem 11 we have that far from the corners with index $j \in \cup_{m=1}^{3} \mathbb{H}_{s^{*}}^{m}$, the optimal control is the projection of a function that is either regular enough or tends to a signed $\infty$ at one point, so it is clear that $\bar{u}_{\text {reg }} \in W^{1-1 / s^{*}, s^{*}}(\Gamma)$.

Next we will check what happens in the neighborhoods of the corners with index $j \in \cup_{m=1}^{3} \mathbb{H}_{s^{*}}^{m}$.

If $0 \notin[a, b]$ then the control would also be flat in neighborhoods of the corners with index $j \in \cup_{m=1}^{3} \mathbb{H}_{s^{*}}^{m}$ again because the normal derivative of the adjoint state is continuous near the corner and 0 at the corner. Then (3.15) holds with $a_{j, m}=0$.

If $a<0<b$, then the optimal control will coincide with $\partial_{n} \bar{\varphi} / \nu$. Using formulas (3.8) and (3.9) and taking into account the definition of the jump functions on the corners $\chi_{j}$ (2.10), we have that there exists $\rho_{j}>0$ such that for all $x \in \Gamma$ such that $\left|x-x_{j}\right|<\rho_{j}$

$$
\bar{u}(x)=\frac{1}{\nu} \partial_{n} \bar{\varphi}(x)=\left\{\begin{array}{cl}
\frac{1}{\nu} \partial_{n} \bar{\varphi}_{r e g}(x)-\frac{\hat{c}_{j, 1} \lambda_{j}}{\nu} r_{j}^{\lambda_{j}-1} & \text { if } j \in \mathbb{H}_{s^{*}}^{1}, \\
\frac{1}{\nu} \partial_{n} \bar{\varphi}_{r e g}(x)-\chi_{j} \frac{\hat{c}_{j, 2} 2 \lambda_{j}}{\nu} r_{j}^{2 \lambda_{j}-1} & \\
-\frac{\hat{c}_{j, 3} 3 \lambda_{j}}{\nu} r_{j}^{3 \lambda_{j}-1} & \text { if } j \in \mathbb{H}_{s^{*}}^{2} \cup \mathbb{H}_{s^{*}}^{3},
\end{array}\right.
$$

and (3.15) holds for $a_{j, m}=-m \hat{c}_{j, m} \lambda_{j} / \nu$.

Let us finally check (3.16). We will write $\bar{y}=y_{1}+y_{2}$, where

$$
-\Delta y_{1}=0 \text { in } \Omega, y_{1}=\bar{u}_{r e g} \text { on } \Gamma
$$

and

$$
-\Delta y_{2}=0 \text { in } \Omega, y_{2}=\sum_{m=1,3} \sum_{j \in \mathbb{H}_{s^{*}}^{m}} a_{j, m} \xi_{j} r^{m \lambda_{j}-1}+\sum_{j \in \mathbb{H}_{s^{*}}^{2}} \chi_{j} a_{j, 2} \xi_{j} r^{2 \lambda_{j}-1} \text { on } \Gamma \text {. }
$$




\section{DIRICHLET CONTROL IN POLYGONAL DOMAINS}

Using Lemma 3 we have that $y_{1} \in W^{1, s}(\Omega)$ for $s \leq s^{*}, s<p_{D}$. From Lemma 5 we have that there exist a unique $y_{2, r e g} \in W^{2, p}(\Omega), p$ defined in (3.6), and unique real numbers $c_{j, m}$ such that

$$
\begin{aligned}
y_{2}= & y_{2, r e g}+\sum_{m=1}^{3} \sum_{j \in \mathbb{H}_{s^{*}}^{m}} a_{j, m} \xi_{j} r_{j}^{m \lambda_{j}-1} s_{j, m}\left(\theta_{j}\right) \\
& +\sum_{m=1}^{3} \sum_{j \in \mathbb{J}_{p}^{m}} c_{j, m} \xi_{j} r_{j}^{m \lambda_{j}} \sin \left(m \theta_{j}\right)
\end{aligned}
$$

Since $p \geq 2$ and $s \leq s^{*}<\infty$, in dimension 2 we have thanks to usual Sobolev's imbedding $W^{2, p}(\Omega) \hookrightarrow W^{1, s}(\Omega)$ that $y_{2, \text { reg }} \in W^{1, s}(\Omega)$.

As we already mentioned, among the terms of the second addend, those which correspond to $\mathbb{H}_{s}^{m}$ are not in $W^{1, s}(\Omega)$. Notice that if $s^{*}<p_{D}$, and hence $s<p_{D}$, then there could be some terms in $W^{1, s}(\Omega)$, which we gather in a function $y_{a, r e g} \in W^{1, s}(\Omega)$.

For the last addend, we have that $\xi_{j} r_{j}^{m \lambda_{j}} \sin \left(m \theta_{j}\right) \notin W^{1, s}(\Omega)$ iff $m \lambda_{j} \leq 1-2 / s$. Since $s \geq 2$, this excludes the case $m>1$. We gather all the other terms in a function $y_{b, r e g} \in W^{1, s}(\Omega)$.

So finally we have that the (3.16) holds for $\bar{y}_{r e g}=y_{1}+y_{2, r e g}+y_{a, r e g}+y_{b, r e g} \in W^{1, s}(\Omega)$ and $c_{j}=c_{j, 1}$.

Let us present now the example announced in Remark 12. For the example, we want to remark that our results are also applicable in curvilinear polygons without many changes (see [10, Th. 5.2.7]). The only thing to take into account is that if the angle $\omega_{j}$ between two curved arcs is grater than $\pi$, then we must impose also $s^{*}<\omega_{j} /\left(\omega_{j}-\pi\right)$ (this is not the case in the following example).

Example 14. Let $\omega_{1}=3 \pi / 2$ and consider the curvilinear polygon $\Omega=\left\{x \in \mathbb{R}^{2}\right.$ : $\left.0<r<1,0<\theta<\omega_{1}\right\}$, where $(r, \theta)$ are the usual polar coordinates. We have that $\omega_{2}=\omega_{3}=\pi / 2$, and hence $\lambda_{1}=2 / 3, \lambda_{2}=\lambda_{3}=2$. Suppose $y_{\Omega} \in L^{\infty}(\Omega)$, so we may choose any $s^{*}<+\infty$. We have $\mathbb{J}_{s^{*}}^{1}=\mathbb{J}_{s^{*}}^{2}=\{1\}$ and $\mathbb{J}_{s^{*}}^{3}=\emptyset$. We also have $p<3$. The adjoint state can be written as

$$
\bar{\varphi}=\bar{\varphi}_{r e g}+\hat{c}_{1,1} \xi_{1} r^{2 / 3} \sin \left(\frac{2}{3} \theta\right)+\chi_{1} \hat{c}_{1,2} \xi_{1} r^{4 / 3} \sin \left(\frac{4}{3} \theta\right)
$$

where $\bar{\varphi}_{\text {reg }} \in W^{2, s^{*}}(\Omega)$ for all $s^{*}<+\infty$. Take $-a=b=1$ for instance. If $\hat{c}_{1,1} \neq 0$, then $\mathbb{H}_{s^{*}}^{m}=\emptyset$ for $m=1,2,3$ and hence $\bar{u}=\bar{u}_{\text {reg }} \in W^{1-1 / s^{*}, s^{*}}(\Gamma)$.

Define now

$$
y_{\Omega}(x)=\left\{\begin{array}{rr}
1 & \text { if } \theta<\omega_{1} / 2 \\
-1 & \text { if } \theta>\omega_{1} / 2
\end{array}\right.
$$

such that the problem is skew-symmetric with respect to the line with $\theta=\omega_{1} / 2$. The skew-symmetry of the data suggests that the solution is skew-symmetric, i. e. that 


\section{DIRICHLET CONTROL IN POLYGONAL DOMAINS}

the symmetric contribution with $r^{2 / 3} \sin \left(\frac{2}{3} \theta\right)$ vanishes, $\hat{c}_{1,1}=0$ (result which we have confirmed numerically), and hence

$$
\bar{u}=\bar{u}_{r e g}+\chi_{1} a_{1,2} \xi_{1} r^{1 / 3}
$$

so $\bar{u} \in W^{1-1 / p, p}(\Gamma)$ for all $p<3$.

In Theorem 13 we have excluded the cases $a=0$ or $b=0$. These cases can be treated with the same techniques. Nevertheless, many cases may appear depending on which of the bounds is zero and the sign of the coefficients of the singular part $\hat{c}_{j, m}$. As an example, we will show how to treat some of these cases. We will discuss first what we think is the "generic" case, and then a seemingly more "rare" case. Without loss of generality suppose $a=0, b>0$.

Case 1 Take $j \in \mathbb{H}_{s *}^{1}$ and suppose $\hat{c}_{j, 1} \neq 0$. Then in the expression for the normal derivative of the adjoint state (3.17), the term $\xi_{j} r_{j}^{\lambda_{j}-1}$ dominates the term $\partial_{n} \varphi_{\text {reg }}$, since $\xi_{j} r^{\lambda_{j}-1} \notin W^{1-1 / s^{*}, s^{*}}(\Gamma)$ and $\partial_{n} \bar{\varphi}_{r e g} \in W^{1-1 / s^{*}, s^{*}}(\Gamma)$. Therefore, if $\hat{c}_{j, 1}<0$, we would have that $\partial_{n} \bar{\varphi}(x) \in[0, b]$ in a neighborhood of $x_{j}$, and hence $\bar{u}(x)$ can be computed as in (3.17). On the other hand, if $\hat{c}_{j, 1}>0$, then $\partial_{n} \bar{\varphi}(x) \leq 0$ in a neighborhood of $x_{j}$, so $\bar{u}(x) \equiv 0$ in that neighborhood. This would be the case of taking Example 14 with the following data: $\omega_{1}=3 \pi / 4, a=0, b=1$ and either $y_{\Omega} \equiv 1$ or $y_{\Omega} \equiv-1$, which would give $\operatorname{sign}\left(c_{j, 1}\right)=-\operatorname{sign}\left(y_{\Omega}\right)$.

Case 2 If $j \in \mathbb{H}_{s^{*}}^{2}$ and $\hat{c}_{j, 2}<0$, then $\partial_{n} \bar{\varphi}(x) \in[0, b]$ in a neighborhood of $x_{j}$ on the side $\Gamma_{j}$, but $\partial_{n} \bar{\varphi}(x) \leq 0$ in a neighborhood of $x_{j}$ on the side $\Gamma_{j-1}$, so on $\Gamma_{j}, \bar{u}(x)$ would have the same expression as in (3.17), but, on $\Gamma_{j-1}, \bar{u}(x)$ would be flat near the corner $x_{j}$. This would be the case of taking Example 14 for $a=0, b=1$ and $y_{\Omega}$ defined in (3.18).

\section{More regular data}

Taking advantage of the regularity of the optimal state, we can obtain several results for more regular data. We will write some results that we think will be useful for the numerical analysis of problem $(\mathrm{P})$. To be specific, to obtain error estimates for problems with regular data, we will need that for $y_{\Omega} \in H^{1}(\Omega), \bar{u} \in H^{3 / 2-\varepsilon}(\Gamma)$ (cf. Corollary 16) and $W^{3, p}(\Omega)$ regularity of the regular part of the adjoint state if $y_{\Omega} \in W^{1, p}(\Omega), p \geq 2$ (see Corollary 17). Some other results can be obtained used the techniques exposed in Section 3 ,

Suppose now that $y_{\Omega} \in H^{t^{*}}(\Omega)$, with $0<t^{*} \leq 1$ such that

$$
\left(1+t^{*}\right) / \lambda_{j} \notin \mathbb{Z} \forall j \in\{1, \ldots, M\} .
$$

For $t>-1$ and $m \in \mathbb{Z}$ define

$$
\tilde{\mathbb{J}}_{t}^{m}=\left\{j \in\{1, \ldots, M\} \text { such that } 0<m \lambda_{j}<1+t\right\}
$$




\section{DIRICHLET CONTROL IN POLYGONAL DOMAINS}

Notice again that due to our choice of $t^{*}$, we only will deal with the cases $m=1,2,3$.

Corollary 15. There exist a unique function $\bar{\varphi}_{r e g} \in H^{2+t^{*}}(\Omega)$ and unique real numbers $\hat{c}_{j, m}$ such that

$$
\bar{\varphi}=\bar{\varphi}_{r e g}+\sum_{m=1}^{3} \sum_{j \in \tilde{\mathbb{J}}_{t^{*}}^{m}} \hat{c}_{j, m} \xi_{j} r_{j}^{m \lambda_{j}} \sin \left(m \lambda_{j} \theta_{j}\right)
$$

Proof. Since $t^{*}>0$, there exists $s^{*}>2$ satisfying (3.4) such that $y_{\Omega} \in L^{s^{*}}(\Omega)$, and hence we can apply Theorem 11 and we have that also $\bar{y} \in W^{1, q}(\Omega) \hookrightarrow H^{1}(\Omega) \hookrightarrow H^{t^{*}}(\Omega)$, where $q>2$ is defined in Theorem 11. The result follows directly from the adjoint state equation (3.3) thanks to the regularity results in [7, §23.C]; see also [11, Th. 2.4.3 and $\S 2.7]$. Notice that due to the conditions imposed on $t^{*}$, logarithmic terms do not appear in the development of the singular part.

To describe the regularity of the optimal control and state, we first introduce the sets $\tilde{\mathbb{H}}_{t}^{m}$ in an analogous way as we did for the sets $\mathbb{H}_{s}^{m}$, the indexes being taken now in the sets $\tilde{\mathbb{J}}_{t}^{m}$ defined above instead of the sets $\mathbb{J}_{s}^{m}$ and considering the coefficients $\hat{c}_{j, 1}$ obtained in Corollary 15. We next define the exponents $t>0$, related to the regularity of the control, and $\tilde{t}>0$, related to the regularity of the state such that

$$
\begin{gathered}
t \leq t^{*}, t<1, t<m \lambda_{j}-1 \text { if } j \in \tilde{\mathbb{H}}_{t}^{m}, m=1,2,3 \\
\tilde{t} \leq t, \tilde{t}<\lambda_{1} .
\end{gathered}
$$

The meaning of these bounds is the following. The regularity of the optimal control will be limited by the regularity of the data, the impossibility of having a control globally in $H^{3 / 2}(\Gamma)$ due to the corners and the bound constraints, and the singular behavior of the control at the convex corners or the "special" nonconvex corners that may lay in $\tilde{\mathbb{H}}_{t}^{m}$ for $m=2,3$. The regularity of the optimal state will be limited by the regularity of the control and the singular behavior at the nonconvex corners of the solution.

Corollary 16. Suppose that

$$
\sharp \partial_{\Gamma}\{x \in \Gamma: \bar{u}(x)=a \text { or } \bar{u}(x)=b\}<+\infty
$$

(the number of points on the boundary in the topology of $\Gamma$ of the active set is finite). Then the optimal control $\bar{u}$ belongs to $H^{1 / 2+t}(\Gamma)$ and $\bar{y} \in H^{1+\tilde{t}}(\Omega)$.

Proof. The proof follows the lines of that of Theorem [11. Since $\bar{\varphi}_{\text {reg }} \in H^{2+t^{*}}(\Omega)$ and $\bar{\varphi}_{\text {reg }}=0$ on $\Gamma$ its normal derivative will be in $H^{1 / 2+t}(\Gamma)$ provided $t \leq t^{*}$ and $t<1$ (the condition $\bar{\varphi}_{\text {reg }}=0$ on $\Gamma$ is needed for $t^{*} \geq 1 / 2$ to prove that the normal derivative tends to zero at the corners, and hence it is continuous; notice that for $t^{*}=1$ this continuity is not enough to have that the normal derivative is in $H^{3 / 2}(\Gamma)$.)

This $H^{1 / 2+t}(\Gamma)$ regularity is not affected by the projection formula (3.1) because $t<1$ and we are supposing (4.1). The same happens with the singular terms such that $\hat{c}_{j, 1} \neq 0$ 


\section{DIRICHLET CONTROL IN POLYGONAL DOMAINS}

and $\lambda_{j}<1$. The rest of the singular terms in the expression for the normal derivative of the adjoint state will be in $H^{1 / 2+t}(\Gamma)$ since $t<m \lambda_{j}-1$ (see equations (3.8) and (3.9) for the expression of the normal derivatives of the singular part.)

Let us prove that $\bar{y} \in H^{1+\tilde{t}}(\Omega)$. Since $\bar{u} \in H^{1 / 2+t}(\Gamma)$, there exists some $U \in H^{1+t}(\Omega)$ such that $U=u$ on $\Gamma$. Moreover, $\Delta U \in H^{-1+t}(\Omega)$. So $z=\bar{y}-U$ is the solution of the boundary value problem

$$
-\Delta z=\Delta U \text { in } \Omega, z=0 \text { on } \Gamma \text {. }
$$

Using the regularity results in [7, §23.C] (see also [11, Th. 2.4.3 and §2.7]) we have that there exist a unique $z_{r e g} \in H^{1+t}(\Omega)$ and unique coefficients $c_{j, m}$ such that

$$
z=z_{r e g}+\sum_{m=1}^{3} \sum_{j \in \tilde{\mathbb{J}}_{-1+t}^{m}} c_{j, m} \xi_{j} r^{m \lambda_{j}} \sin \left(m \lambda_{j} \theta_{j}\right) .
$$

Since $\tilde{t}<\lambda_{1}$, the singular part is in $H^{1+\tilde{t}}(\Omega)$, and so is the optimal state.

Finally, we will describe the adjoint state for even more regular data. In the rest of this section we will suppose $y_{\Omega} \in W^{1, p^{*}}(\Omega), p^{*} \geq 2$.

For $p>1$ and $m \in \mathbb{Z}$ define

$$
\mathbb{J}_{1, p}^{m}=\left\{j \in\{1, \ldots, M\} \text { such that } 0<m \lambda_{j}<3-\frac{2}{p} \text { and } m \lambda_{j} \notin \mathbb{Z}\right\} .
$$

Now we have that $\mathbb{J}_{1, p}^{m}=\emptyset$ if $m>5$. We have to add the condition $m \lambda_{j} \notin \mathbb{Z}$ otherwise logarithmic terms may appear. Define also:

$$
\mathbb{L}_{1, p}^{m}=\left\{j \in\{1, \ldots, M\} \text { such that } 0<m \lambda_{j}<3-\frac{2}{p} \text { and } m \lambda_{j} \in \mathbb{Z}\right\} .
$$

A direct calculation gives us that $\mathbb{L}_{1, p}^{m}=\emptyset$ if $m=2$ or $m \geq 4, \mathbb{L}_{1, p}^{1} \subset\left\{j: \omega_{j}=\pi / 2\right\}$ and $\mathbb{L}_{1, p}^{3} \subset\left\{j: \omega_{j}=3 \pi / 2\right\}$, and hence $m \lambda_{j}=2$ if $j \in \mathbb{L}_{1, p}^{m}$.

Consider now $p \geq 2$ such that, for $m=1,2,3$

$$
p \leq p^{*}, p<p_{D}, p<\frac{2}{2-m \lambda_{j}} \text { if } j \in \mathbb{H}_{s^{*}}^{m} \forall s^{*}<\infty .
$$

In addition, we need to to assume

$$
\frac{3 p-2}{\lambda_{j} p} \notin \mathbb{Z} \forall j \in\{1, \ldots, M\} .
$$

With this notation, we have the following result. 


\section{DIRICHLET CONTROL IN POLYGONAL DOMAINS}

Corollary 17. There exist a unique function $\bar{\varphi}_{\text {reg }} \in W^{3, p}(\Omega)$ and unique real numbers $\hat{c}_{j, m}$ and $\hat{d}_{j, m}$ such that

$$
\begin{aligned}
\bar{\varphi}= & \bar{\varphi}_{r e g}+\sum_{m=1}^{5} \sum_{j \in \mathbb{J}_{1, p}^{m}} \hat{c}_{j, m} \xi_{j} r_{j}^{m \lambda_{j}} \sin \left(m \lambda_{j} \theta_{j}\right) \\
& +\sum_{m=1,3} \sum_{j \in \mathbb{L}_{1, p}^{m}} \hat{d}_{j, m} \xi_{j} r_{j}^{2}\left(\log \left(r_{j}\right) \sin \left(2 \theta_{j}\right)+\theta_{j} \cos \left(2 \theta_{j}\right)\right) .
\end{aligned}
$$

Proof. Since $p^{*} \geq 2, y_{\Omega} \in L^{s^{*}}(\Omega)$ for any $s^{*}<\infty$, and hence we can apply Theorem 11 and we have that also $\bar{y} \in W^{1, p}(\Omega)$. The result follows directly from the adjoint state equation (3.3) thanks to the regularity result [10, Th. 5.1.3.5]

$$
\begin{aligned}
\bar{\varphi}= & \bar{\varphi}_{r e g}+\sum_{m=1}^{5} \sum_{j \in \mathbb{J}_{1, p}^{m}} \hat{\xi}_{j} r_{j}^{m \lambda_{j}} \sin \left(m \lambda_{j} \theta_{j}\right) \\
& +\sum_{m=1,3} \sum_{j \in \mathbb{L}_{1, p}^{m}} \hat{d}_{j, m} \xi_{j} r_{j}^{m \lambda_{j}}\left(\log \left(r_{j}\right) \sin \left(m \lambda_{j} \theta_{j}\right)+\theta_{j} \cos \left(m \lambda_{j} \theta_{j}\right)\right) .
\end{aligned}
$$

and using that $m \lambda_{j}=2$ if $j \in \mathbb{L}_{1, p}^{m}$.

\section{Problems without control constraints}

For problems without control constraints, we obtain similar results. Indeed, for convex domains and data $y_{\Omega} \in L^{s^{*}}(\Omega), s^{*}>2$, it is obvious from Theorem 18 below that the optimal control is a bounded function and hence all the results stated before apply. Nevertheless, for nonconvex domains, we will not obtain a continuous control and the singularities must be taken into account near all the corners. Therefore, the indexes for the expansion of the singular parts must be taken running through all the sets $\mathbb{J}_{s}^{m}$, and not only through the sets $\mathbb{H}_{s}^{m}$.

Theorem 18. Suppose now that $-a=b=\infty$ and $y_{\Omega} \in L^{s^{*}}(\Omega), s^{*} \geq 2$. Then there exists a unique $\bar{\varphi}_{r e g} \in W^{2, s}(\Omega), \bar{u}_{r e g} \in W^{1-1 / s, s}(\Gamma), \bar{y}_{r e g} \in W^{1, s}(\Omega)$, for all $s \leq s^{*}$, $s<p_{D}$, and unique real numbers $\left(\hat{c}_{j, m}\right)_{j \in \mathbb{J}_{s}^{m}}$ and $\left(c_{j}\right)_{\lambda_{j}<1-2 / s}$ such that

$$
\begin{gathered}
\bar{\varphi}=\bar{\varphi}_{r e g}+\sum_{m=1}^{3} \sum_{j \in \mathbb{J}_{s}^{m}} \hat{c}_{j, m} r_{j}^{m \lambda_{j}} \sin \left(m \lambda_{j} \theta_{j}\right) \xi_{j} \\
\bar{u}(x)=\bar{u}_{r e g}+\sum_{m=1,3} \sum_{j \in \mathbb{J}_{s}^{m}} a_{j, m} \xi_{j} r^{m \lambda_{j}-1}+\sum_{j \in \mathbb{J}_{s}^{2}} \chi_{j} a_{j, m} \xi_{j} r^{2 \lambda_{j}-1} \\
\bar{y}=\bar{y}_{r e g}+\sum_{m=1}^{3} \sum_{j \in \mathbb{J}_{s}^{m}} a_{j, m} \xi_{j} r_{j}^{m \lambda_{j}-1} s_{j, m}\left(\theta_{j}\right)+\sum_{\lambda_{j}<1-2 / s} c_{j} \xi_{j} r_{j}^{\lambda_{j}} \sin \left(\lambda_{j} \theta_{j}\right)
\end{gathered}
$$




\section{DIRICHLET CONTROL IN POLYGONAL DOMAINS}

where $a_{j, m}$ and $s_{j, m}(\theta)$ are given by the formulas (3.13) and (3.14).

Proof. The proof is very similar to those of theorems 9, 11 and 13. We will only emphasize on the main difference: at the beginning of the proof of Theorem 9 we used that the optimal control was bounded to obtain that the optimal state was a function in $L^{s^{*}}(\Omega)$. Now the optimal control is not bounded, so we use a bootstrapping argument to show that $\bar{y} \in L^{s}(\Omega)$ for $s \leq s^{*}, s<p_{D}$. The result follows then using the same techniques as before. Notice that now we do not need the sets $\mathbb{H}_{s}^{m}$, since we do not have to exclude in the expression of the singular part of the control the corners where the normal derivative of the adjoint state is not bounded.

In a first step we have that $\bar{u} \in L^{2}(\Gamma)$, and hence $\bar{y} \in H^{1 / 2}(\Omega) \subset L^{4}(\Omega)$. If $s^{*} \leq 4$ the proof is complete since $4<p_{D}$ for any polygonal domain.

Suppose $s^{*}>4$. The normal derivative of the regular part of the adjoint state is in $W^{1-1 / 4,4}(\Gamma)$, but now, since we have no control constraints, we have to take into account the normal derivative of the singular part near the non-convex corners. We have so far that the optimal control can be written as the sum of a regular part, which is in $W^{1-1 / 4,4}(\Gamma)$ plus a singular part, that behaves as $r_{1}^{\lambda_{1}-1}$. For the regular part we apply Lemma 3 and for the singular part we apply Lemma 5 , and we have that the optimal state can be written as the sum of a regular part which is in $W^{1,4}(\Omega) \subset L^{s^{*}}(\Omega)$ plus a singular part that behaves at worst as $r_{1}^{\lambda_{1}-1} \xi_{1} \in L^{s}(\Omega)$ for all $s<2 /\left(1-\min \left\{1, \lambda_{1}\right\}\right)=p_{D}$. So we have that $\bar{y} \in L^{s}(\Omega)$ for all $s \leq s^{*}, s<p_{D}$.

We will finish this section stating some regularity results of the optimal solution in the unconstrained case in some special situations.

Corollary 19. Suppose the assumptions of Theorem 18 are satisfied. Then, for all $p \leq s^{*}, p<p_{\Omega}$ we have that $\bar{\varphi} \in W^{2, p}(\Omega), \bar{u} \in W^{1-1 / p, p}(\Gamma)$ and $\bar{y} \in W^{1, p}(\Omega)$.

Proof. Since $p \leq s^{*}$ and $p<p_{\Omega}<p_{D}$, the regular parts of the involved functions satisfy $\bar{u}_{r e g} \in W^{1-1 / p, p}(\Gamma), \bar{y}_{r e g} \in W^{1, p}(\Omega)$ and $\bar{\varphi}_{r e g} \in W^{2, s}(\Omega)$ due to Theorem 18.

On the other hand, the assumption $p<p_{\Omega}$ implies $\xi_{1} r_{1}^{\lambda_{1}-1} \in W^{1, p}(\Omega)$, and hence obviously $\xi_{1} r_{1}^{\lambda_{1}} \in W^{2, p}(\Omega)$ and $\xi_{1} r_{1}^{\lambda_{1}-1} \in W^{1-1 / p, p}(\Gamma)$. Since these are the worst terms we may find in the singular parts, the proof is complete.

With the same techniques of Section 4, (Corollary 15] and 16) we can obtain the following result.

Corollary 20. Suppose the assumptions of Theorem 18 are satisfied and $y_{\Omega} \in H^{t^{*}}(\Omega)$ for some $t^{*} \leq 1$. Define $t>0$ such that

$$
t \leq t^{*}, t<1, t<\lambda_{1}-1 \text {. }
$$

Then $\bar{u} \in H^{1 / 2+t}(\Gamma)$ and $\bar{y} \in H^{1+t}(\Omega)$. 


\section{DIRICHLET CONTROL IN POLYGONAL DOMAINS}

\section{References}

[1] Th. Apel, S. Nicaise, And J. Pfefferer, Discretization of the Poisson equation with non-smooth data and emphasis on non-convex domains. Preprint arXiv:1505.01229, 2015.

[2] M. Bergaren, Approximations of very weak solutions to boundary-value problems, SIAM J. Numer. Anal., 42 (2004), pp. 860-877 (electronic).

[3] S.C. Brenner And L.R. Scott, The Mathematical Theory of Finite Element Methods, Springer-Verlag, New York, Berlin, Heidelberg, 1994.

[4] E. Casas, A. Günther, And M. Mateos, A paradox in the approximation of Dirichlet control problems in curved domains, SIAM J. Control Optim., 49 (2011), pp. 1998-2007.

[5] E. Casas, M. Mateos, and J.-P. Raymond, Penalization of Dirichlet optimal control problems, ESAIM Control Optim. Calc. Var., 15 (2009), pp. 782-809.

[6] E. CASAS AND J.-P. RAYMOND, Error estimates for the numerical approximation of Dirichlet boundary control for semilinear elliptic equations, SIAM J. Control Optim., 45 (2006), pp. 1586-1611 (electronic).

[7] M. Dauge, Elliptic Boundary Value Problems in Corner Domains, vol. 1341 of Lecture Notes in Mathematics, Springer-Verlag, Berlin, 1988.

[8] — Neumann and mixed problems on curvilinear polyhedra, Integr. Equat. Oper. Th., 15 (1992), pp. 227-261.

[9] K. Deckelnick, A. Günther, And M. Hinze, Finite element approximation of Dirichlet boundary control for elliptic PDEs on two- and three-dimensional curved domains, SIAM J. Control Optim., 48 (2009), pp. 2798-2819.

[10] P. Grisvard, Elliptic problems in nonsmooth domains, vol. 24 of Monographs and Studies in Mathematics, Pitman (Advanced Publishing Program), Boston, MA, 1985.

[11] —, Singularities in boundary value problems, vol. 22 of Recherches en Mathématiques Appliquées [Research in Applied Mathematics], Masson, Paris; Springer-Verlag, Berlin, 1992.

[12] D. Jerison And C.E. Kenig, The inhomogeneous Dirichlet problem in Lipschitz domains, J. Funct. Anal., 130 (1995), pp. 161-219.

[13] J.L. Lions and E. Magenes, Problèmes aux Limites non Homogènes, Dunod, Paris, 1968.

[14] S. May, R. RAnnacher, And B. Vexler, Error analysis for a finite element approximation of elliptic Dirichlet boundary control problems, SIAM Journal on Control and Optimization, 51 (2013), pp. 2585-2611.

[15] G. Of, T.X. Phan, and O. Steinbach, Boundary element methods for Dirichlet boundary control problems, Mathematical Methods in the Applied Sciences, 33 


\section{DIRICHLET CONTROL IN POLYGONAL DOMAINS}

(2010), pp. 2187-2205.

[16] Guido Stampacchia, Le problème de Dirichlet pour les équations elliptiques du second ordre à coefficients discontinus, Ann. Inst. Fourier (Grenoble), 15 (1965), pp. 189-258. 